\title{
ON THE CATEGORY OF CERTAIN CLASSES OF TRANSFORMATIONS IN ERGODIC THEORY $\left({ }^{1}\right)$
}

\author{
BY \\ A. IONESCU TULCEA
}

The main purpose of this paper is to establish some category theorems for certain classes of "invertible measurable and nonsingular transformations" on the unit interval. We chose our setting to be that of the unit interval in order to simplify the presentation $\left({ }^{2}\right)$. The paper is divided into two parts. The first part contains the background material; here we make precise our terminology and prove some preliminary results. The main result of Part I is an approximation theorem (Theorem 1) which is used essentially in the second part of the paper, but which may be of independent interest. In Part II we prove a category theorem for the positive invertible isometries of $L^{1}$ (Theorem 2); from this in turn we derive our main conclusions (Theorem 3). We wish to remark here that the proofs of our theorems are straightforward and do not use, at any stage, the existence of the concrete examples constructed by D. S. Ornstein in [10] and by R. V. Chacon in [2]. From this point of view our paper is independent of [10] and [2]. In a certain sense also, Theorem 3 of this paper unifies and extends the results of [10] and [2]. We wish to add, however, that we were largely inspired by [10], [2] and [3] and wish to acknowledge our indebtedness to the ideas contained in these papers.

The principal results of this paper were announced in [7].

\section{PART I}

1. Let $X=[0,1], \mathscr{B}$ the $\sigma$-algebra of Lebesgue measurable subsets of $X$, $\mu$ the Lebesgue measure. Let $\mathscr{S}$ be the vector space of all real-valued $\mathscr{B}$ measurable functions on $X ; \mathscr{S}$ is endowed with the semi-distance

$$
(f, g) \rightarrow \rho(f, g)=\int_{X} \frac{|f-g|}{1+|f-g|} d \mu .
$$

As is well known (see $[4$, p. 178]), "convergence with respect to $\rho$ " is equivalent to "convergence in measure." As usual, we shall denote with $\mathscr{L}^{\infty}$ the vector space of all $f \in \mathscr{S}$ that are essentially bounded and with $\mathscr{L}^{1}$ the vector space of all $f \in \mathscr{S}$ that are Lebesgue integrable; $\mathscr{L}^{1}$ is endowed with the

Received by the editors August 12, 1963.

( $\left.{ }^{1}\right)$ Research supported by the U. S. Army Research Office (Durham) under contract DA -ARO(D)-31-124-G218.

$\left({ }^{2}\right)$ The extension to more general cases does not present difficulty and is left to the interested reader. 
semi-norm $f \rightarrow\|f\|=S_{X}|f| d \mu$. Denote by $L^{1}$ the associated Banach space and by $f \rightarrow f$ the canonical mapping of $\mathscr{L}^{1}$ onto $L^{1}$.

To simplify our notation, we shall use the term "automorphism" for an "invertible measurable and nonsingular transformation" on the unit interval. Specifically, we shall call automorphism any bijective mapping $\tau$ of $X$ onto $X$ satisfying the following conditions:

(i) if $E \in \mathscr{B}$, then $\tau(E) \in \mathscr{B}$ and $\tau^{-1}(E) \in \mathscr{B}$;

(ii) if $A \in \mathscr{B}$ and $\mu(A)=0$, then $\mu(\tau(A))=\mu\left(\tau^{-1}(A)\right)=0$.

The set $\mathscr{A}$ of all automorphisms is a group for the usual composition $\left(\tau_{1}, \tau_{2}\right) \rightarrow \tau_{1} \circ \tau_{2}$; we shall denote with $e$ the unit element of this group. For $\tau_{1}, \tau_{2} \in \mathscr{L}$ we shall write $\tau_{1} \equiv \tau_{2}$ if $\mu\left(\left\{x \mid \tau_{1}(x) \neq \tau_{2}(x)\right\}\right)=0$; this defines an equivalence relation $R$ in $\mathscr{A}$ compatible with the group structure of $\mathscr{A}$. Denote by $\tau \rightarrow \tilde{\tau}$ the canonical mapping of $\mathscr{A}$ onto the quotient group $\mathscr{A} / R$.

Let now $\mathscr{L}\left(L^{1}, L^{1}\right)$ be the algebra of all continuous linear mappings of $L^{1}$ into $L^{1}$. For $T \in \mathscr{L}\left(L^{1}, L^{1}\right)$ and $\widetilde{f} \in L^{1}$ we shall denote by $T f$ a representative of the class $T \tilde{f}$. Let $\mathscr{G}$ be the set of all positive invertible isometries of $L^{1}$, that is the set of all $T \in \mathscr{L}_{\widetilde{\sim}}\left(L_{\sim}^{1}, L_{\widetilde{\sim}}^{1}\right)$ such that: $(j)\|T \widetilde{f}\|=\|\widetilde{f}\|$ for each $\widetilde{f} \in L^{1} ;(\mathrm{jj}) T\left(L^{1}\right)=L^{1} ;(\mathrm{jjj}) T \widetilde{f} \geqq \widetilde{0}$ if $\widetilde{f} \in L^{1}$ and $\widetilde{f} \geqq \widetilde{0}$.

The set $\mathscr{G}$ is a multiplicative group; we shall denote by $I$ the identity operator (= the unit element of $\mathscr{G}$ ).

For every $\tau \in \mathscr{A}$ we shall denote by $\mu \circ \tau^{-1}$ the measure on $\mathscr{B}$ defined by the equations $\left(\mu \circ \tau^{-1}\right)(E)=\mu\left(\tau^{-1}(E)\right), E \in \mathscr{B}$. The measure $\mu \circ \tau^{-1}$ is clearly absolutely continuous with respect to $\mu$ (in fact even equivalent to $\mu$ ); we shall denote by $\left(d_{\mu} \circ \tau^{-1}\right) / d_{\mu}$ the Radon-Nikodym derivative of $\mu^{\circ} \tau^{-1}$ with respect to $\mu$. For every $\tau \in \mathscr{A}$ we shall denote by $T_{\tau}$ the isometry induced by $\tau ; T_{\tau}$ is defined by the equations:

$$
T_{\tau} f=f \circ \tau^{-1} \cdot \frac{d \mu \circ \tau^{-1}}{d \mu}, \quad f \in \mathscr{L}^{1} .
$$

It is easily seen that $T_{\tau} \in \mathscr{G}$. By a theorem of S. Banach (see [1, p. 178] and [9]) every $T \in \mathscr{G}$ is induced in the above sense by some automorphism $\tau$; it follows that the mapping $\tau \rightarrow T_{\tau}$ is a representation of the group $\mathscr{A}$ onto the group $\mathscr{G}$. Now for $\tau_{1}, \tau_{2} \in \mathscr{A}$ we have $T_{\tau_{1}}=T_{\tau_{2}}$ if and only $\tau_{1} \equiv \tau_{2}$. We deduce that the mapping $\tilde{\tau} \rightarrow T_{\tau}$ is an isomorphism of $\mathscr{A} / R$ onto $\mathscr{G}$.

2. In what follows, the term partition (respectively, almost partition) of $X$ will always mean a finite collection say $\left\{A_{1}, \cdots, A_{p}\right\}$ of pairwise disjoint sets such that: (i) $A_{k} \in \mathscr{B}, \mu\left(A_{k}\right)>0$ for each $1 \leqq k \leqq p$; (ii) $\bigcup_{1 \leqq k \leqq p} A_{k}=X$ (respectively, $\mu\left(\bigcup_{1 \leqq k \leqq p} A_{k}\right)=1$ ).

Given a set $Y \in \mathscr{B}$ with $\mu(Y)>0$ and an almost partition $\Delta$ of $X$, we shall denote with $\Delta \cap Y$ the "almost partition of $Y$ " induced by $\Delta$; this is obtained by excluding the sets of measure zero from the collection $\{A \cap Y \mid A \in \Delta\}$. 
Let $B \in \mathscr{B}$ and define the symbol $\delta B$ to mean $B$ if $\delta=1$ and $C B$ if $\delta=0$. Given $B_{1}, \cdots, B_{m} \in \mathscr{B}$, the almost partition of $X$ generated by $B_{1}, \cdots, B_{m}$ is obtained by excluding the sets of measure zero from the collection of all sets of the form $\bigcap_{1 \leqq i \leqq m} \delta_{i} B_{i}$, where $\delta_{i} \in\{0,1\}$ for $1 \leqq i \leqq m$.

Given two almost partitions of $X, \Delta_{1}$ and $\Delta_{2}$, we say that $\Delta_{2}$ is finer than $\Delta_{1}$ if for every $B \in \Delta_{2}$ there is $A \in \Delta_{1}$ such that $B \subset A$.

If $\Delta^{\prime}, \Delta^{\prime \prime}$ are two almost partitions of $X$, we shall use the notation $\Delta^{\prime} \vee \Delta^{\prime \prime}$ for the almost partition of $X$ obtained by excluding the sets of measure zero from the collection $\left\{A \cap B \mid A \in \Delta^{\prime}, B \in \Delta^{\prime \prime}\right\}$.

If $\tau \in \mathscr{A}$ and $\Delta$ is an almost partition of $X$, we shall denote with $\tau \Delta$ the almost partition $\{\tau(A) \mid A \in \Delta\}$.

We shall give below several definitions. It will be convenient, for the purposes of the present paper, to modify slightly the terminology of the note [7].

Let $\quad \tau \in \mathscr{A}, \quad k \in N^{*}=\{1,2,3, \cdots\} ; \quad$ let $\quad E_{1} \in \mathscr{B}, \ldots, E_{k} \in \mathscr{B}$ and $n_{1} \in N^{*}, \cdots, n_{k} \in N^{*}$.

Definition 1. We say that $\left(E_{1}, \ldots, E_{k}\right)$ is an admissible system of order $\left(n_{1}, \cdots, n_{k}\right)$ for $\tau$ if: (1) the sequence $\left(\tau^{j}\left(E_{i}\right)\right)_{1 \leqq i \leqq k, 0 \leqq j \leqq n_{i}-1}$ constitutes an almost partition of $X$ (we shall denote this almost partition with $\left.\Delta\left(\tau ;\left(E_{1}, \cdots, E_{k}\right) ;\left(n_{1}, \cdots, n_{k}\right)\right)\right) ;(2)$ for each $1 \leqq i \leqq k$ and each $x \in E_{i}$, the sequence $\left(\tau^{j}(x)\right)_{0 \leqq j<\infty}$ is periodic with strict period $\left(^{3}\right) n_{i}$.

Definition 2. We say that $\left(E_{1}, \cdots, E_{k}\right)$ is an s-admissible system of order $\left(n_{1}, \cdots, n_{k}\right)$ for $\tau$ if: (1) $\left(E_{1}, \cdots, E_{k}\right)$ is an admissible system of order $\left(n_{1}, \ldots, n_{k}\right)$ for $\tau$; (2) $\left(d \mu \circ \tau^{-1}\right) / d_{\mu}$ is constant (almost everywhere) on each of the sets of the partition $\Delta\left(\tau ;\left(E_{1}, \cdots, E_{k}\right) ;\left(n_{1}, \cdots, n_{k}\right)\right)$.

If $k=1$ and $n=n_{1}$ we shall simply speak of an admissible (or $s$-admissible) set of order $n$ for $\tau$.

With this terminology, if $E$ is an admissible set of order $n$ for $\tau$, we set

$$
\delta(E, \tau)=\sup _{0 \leqq j \leqq n-1} \mu\left(\tau^{j}(E)\right) .
$$

We recall now the following result due to $\mathrm{C}$. E. Linderholm:

(A) Let $Y \subset X$ be a Lebesgue measurable set with $\mu(Y)>0$ and let $\zeta$ be an automorphism of $Y\left({ }^{4}\right)$. Assume that there is $n \in N^{*}$ such that for every $y \in Y$ the sequence $\left(\zeta^{j}(y)\right)_{0 \leq j<\infty}$ is periodic with strict period $n$. There is then an admissible set $\left({ }^{4}\right)$ of order $n$ for $\zeta$.

For completeness and since we need this result later, we sketch a proof

$\left(^{3}\right)$ Let $A$ be a set, $\left(a_{j}\right)_{0 \leqq j<\infty}$ a sequence of elements of $A$. The sequence $\left(a_{j}\right)_{0 \leqq j<\infty}$ is penodic if there is $n \in N^{*}$ such that $a_{j+n}=a_{j}$ for all $0 \leqq j<\infty$. The smallest integer $n$ with this property is called the strict period of the sequence $\left(a_{j}\right)_{0 \leqq j<\infty}$.

$\left({ }^{4}\right)$ The notions of automorphism, admissible and $s$-admissible system make sense equally well for an arbitrary measure space. We shall use these notions, without further explanation, in the case when the underlying space is $Y$ with the induced measure space structure $\left(Y, \mathscr{D}_{Y}, \mu_{Y}\right)$ (here, $\mathscr{B}_{Y}=\mathscr{D} \cap Y$ and $\mu_{Y}$ is the restriction of $\mu$ to $\mathscr{B}_{Y}$, i.e., $\mu_{Y}(E)=\mu(E)$ for $E \in \mathscr{B}_{Y}$ ). 
below (see also $[5$, p. 70$]$ and $[8$, p. 117$]$ for the analogy with the measurepreserving case).

We may assume that $n>1$ (for $n=1$ the proposition is obvious). We shall show first that:

$(\alpha)$ Given any $E \in \mathscr{B}_{Y}$ with $\mu(E)>0$ and any $1 \leqq j \leqq n-1$, there is $F \subset E$, $F \in \mathscr{B}_{Y}, \mu(F)>0$ such that $\mu\left(F \triangle \zeta^{j}(F)\right)>0$.

Suppose that $(\alpha)$ is not true. There is then $E \in \mathscr{B}_{Y}$ with $\mu(E)>0$ and $1 \leqq i \leqq n-1$ such that the relations $F \subset E, F \in \mathscr{B}_{Y}, \mu(F)>0$ imply $\mu\left(F \triangle \zeta^{i}(F)\right)=0$ and hence $\mu\left(F \triangle \zeta^{-i}(F)\right)=0$. In. particular, $\mu\left(E \triangle \zeta^{-i}(E)\right)$ $=0$. Let now $A=\left\{x \in E \mid \zeta^{i}(x) \neq x\right\}$. Let $\left(D_{p}\right)_{1 \leqq p<\infty}$ be a sequence of sets belonging to $\mathscr{B}_{Y}$ and separating the points of $Y$. Then

$$
A=E \cap\left(\bigcup_{1 \leqq p<\infty} D_{p} \Delta \zeta^{-i}\left(D_{p}\right)\right)=\bigcup_{1 \leqq p<\infty}\left(E \cap D_{p}\right) \Delta\left(E \cap \zeta^{-i}\left(D_{p}\right)\right)
$$

and the above considerations imply that $\mu(A)=0$. This contradicts the hypothesis of the proposition; therefore $(\alpha)$ is proved.

We shall show now that:

$(\beta)$ Given any $G \in \mathscr{B}_{Y}$ with $\mu(G)>0$, there is $H \subset G, H \in \mathscr{B}_{Y}, \mu(H)>0$ such that the sets of the sequence $\left(\zeta^{j}(H)\right)_{0 \leqq j \leqq n-1}$ are pairwise disjoint.

Remark first that the hypothesis of the proposition implies that $\zeta^{j}$ is conservative (see $\left[5\right.$, p. 11]) for each $1 \leqq j \leqq n-1$. Let now $G \in \mathscr{B}_{Y}$ with $\mu(G)>0$. By $(\alpha)$ there is $F_{1} \subset G, F_{1} \in \mathscr{B}_{Y}, \mu\left(F_{1}\right)>0$ such that $\mu\left(F_{1} \triangle \zeta\left(F_{1}\right)\right)$ $>0$. Since $\zeta$ is conservative, $\mu\left(F_{1}-\zeta\left(F_{1}\right)\right)>0$. Define $H_{1}=F_{1}-\zeta\left(F_{1}\right)$; then $H_{1} \subset G, \mu\left(H_{1}\right)>0$ and $H_{1} \cap \zeta\left(H_{1}\right)=\varnothing$. If $n=2$ we set $H=H_{1}$ and the proof of $(\beta)$ is finished.

If $n>2$ we proceed by induction. Suppose that for some $1 \leqq k<n-1$ we have constructed a set $H_{k} \subset G, H_{k} \in \mathscr{B}_{Y}, \mu\left(H_{k}\right)>0$ such that the sets of the sequence $\left(\zeta^{j}\left(H_{k}\right)\right)_{0 \leqq j \leqq k}$ are pairwise disjoint. Applying $(\alpha)$ we find a set $F_{k+1} \subset H_{k}, F_{k+1} \in \mathscr{B}_{Y}, \mu\left(F_{k+1}\right)>0$ such that $\mu\left(F_{k+1} \triangle \zeta^{k+1}\left(F_{k+1}\right)\right)>0$. Since $\zeta^{k+1}$ is conservative, $\mu\left(F_{k+1}-\zeta^{k+1}\left(F_{k+1}\right)\right)>0$. Define $H_{k+1}=F_{k+1}-\zeta^{k+1}\left(F_{k+1}\right)$; we thus obtain a set $H_{k+1} \subset G, H_{k+1} \in \mathscr{B}_{Y}, \mu\left(H_{k+1}\right)>0$ such that the sets of the sequence $\left(\zeta^{j}\left(H_{k+1}\right)\right)_{0 \leqq j \leqq k+1}$ are pairwise disjoint. This completes the proof of $(\beta)$.

( $\gamma)$ There is an admissible set of order $n$ for $\zeta$. Let $\mathscr{F}=\left\{\widetilde{B}\left({ }^{5}\right) \mid B \in \mathscr{B}_{\mathrm{Y}}\right.$, $\mu(B)>0$, the sets of the sequence $\left(\zeta^{j}(B)\right)_{0 \leqq j \leqq n-1}$ are pairwise disjoint $\}$. By $(\beta), \mathscr{F}$ is nonvoid. Now it is easily seen that $\mathscr{F}$ is an inductive set for the natural order relation. Let $\widetilde{B}_{0} \in \mathscr{F}$ be a maximal element; $B_{0}$ must then be an admissible set of order $n$ for $\zeta$. In fact, otherwise $\mu\left(Y-\left(\bigcup_{0 \leqq j \leqq n-1} \zeta^{j}\left(B_{0}\right)\right)\right)$ $>0$; letting $G=Y-\left(\bigcup_{0 \leqq j \leqq n-1} \zeta^{j}\left(B_{0}\right)\right)$, we note that $G \in \mathscr{B}_{Y}, \mu(G)>0$ and

$\left({ }^{5}\right)$ For $E \in \mathscr{B}, F \in \mathscr{B}$ we write $E \equiv F$ if $\mu(E \Delta F)=0$. This defines an equivalence relation in $\mathscr{B}$. For each $E \in \mathscr{B}$ we denote with $\widetilde{E}$ the corresponding equivalence class. 
$\zeta(G)=G$. By $(\beta)$, there is $B_{1} \subset G, B_{1} \in \mathscr{B}_{Y}, \mu\left(B_{1}\right)>0$ such that the sets of the sequence $\left(\zeta^{j}\left(B_{1}\right)\right)_{0 \leqq j \leqq n-1}$ are pairwise disjoint. But then if $\mathbb{Z}^{\prime}=B_{0} \cup B_{1}$, we have $\widetilde{B}^{\prime} \in \mathscr{F}$ and $\widetilde{B}_{0}<\widetilde{B}^{\prime}$ contradicting the maximality of $\widetilde{B}_{0}$. This completes the proof of the proposition.

From proposition (A) we deduce:

(B) Let $\tau \in \mathscr{A}$ and assume that there is $n \in N^{*}$ such that $\tau^{n} \equiv e$. There are then $E_{1} \in \mathscr{B}, \cdots, E_{k} \in \mathscr{B}$ and $n_{1} \in N^{*}, \cdots, n_{k} \in N^{*}$ such that $\left(E_{1}, \cdots, E_{k}\right)$ is an admissible system of $\operatorname{order}\left(n_{1}, \cdots, n_{k}\right)$ for $\tau$.

For each $1 \leqq p<\infty$ let $Y_{p}$ be the set of all $x \in X$ such that the sequence $\left(\tau^{s}(x)\right)_{0 \leqq s<\infty}$ is periodic with strict period $p$; note that $Y_{p} \in \mathscr{B}$ and $\tau\left(Y_{p}\right)=Y_{p}$. The hypothesis of our proposition implies that there are integers $1 \leqq n_{1}<$ $\cdots<n_{k} \leqq n$ such that the corresponding sequence $\left(Y_{n_{i}}\right)_{1 \leqq i \leqq k}$ constitutes an almost partition of $X$. Now it is enough to apply proposition (A) to the set $Y_{n_{i}}$ and to $\tau_{\mid Y_{n_{j}}}$ (=the restriction of $\tau$ to $Y_{n_{i}}$ ) for each $1 \leqq i \leqq k$.

Definition 3. An automorphism $\tau$ will be called periodic if there is $n \in N^{*}$ such that $\tau^{n} \equiv e$.

We shall denote $\mathscr{P}=\left\{T=T_{\tau} \in \mathscr{G} \mid \tau\right.$ is periodic $\}$. It is obvious that for $T \in \mathscr{G}$ we have $T \in \mathscr{P}$ if and only if $T^{n}=I$ for some $n \in N^{*}$.

By proposition (B) above, if $\tau \in \mathscr{A}$ is periodic, there are sets $E_{1} \in \mathscr{B}$, $\cdots, E_{k} \in \mathscr{B}$ and integers $n_{1} \in N^{*}, \cdots, n_{k} \in N^{*}$ such that $\left(E_{1}, \cdots, E_{k}\right)$ is an admissible system of order $\left(n_{1}, \cdots, n_{k}\right)$ for $\tau$.

Definition 4. An automorphism $\tau$ will be called strictly periodic if there is $n \in N^{*}$ such that for almost every $x \in X$, the sequence $\left(\tau^{s}(x)\right)_{0 \leqq s<\infty}$ is periodic with strict period $n$. The integer $n$ will be called the strict period of $\tau$.

We shall denote $\mathscr{P}_{1}=\left\{T=T_{\tau} \in \mathscr{G} \mid \tau\right.$ is strictly periodic $\}$.

We have obviously $\mathscr{P}_{1} \subset \mathscr{P}$.

By proposition (A) above, if $\tau \in \mathscr{A}$ is strictly periodic with strict period $n$, there is a set $E \in \mathscr{B}$ admissible of order $n$ for $\tau$.

The above considerations suggest the introduction of the following two classes of isometries:

We shall denote with $\mathscr{Q}$ the set of all $T=T_{\tau} \in \mathscr{G}$ such that: (1) $\tau$ is periodic, and (2) there are sets $E_{1} \in \mathscr{B}, \cdots, E_{k} \in \mathscr{B}$ and integers $n_{1} \in N^{*}$, $\cdots, n_{k} \in N^{*}$ such that $\left(E_{1}, \ldots, E_{k}\right)$ is an $s$-admissible system of order $\left(n_{1}, \cdots, n_{k}\right)$ for $\tau$. We have obviously $\mathscr{Q} \subset \mathscr{P}$.

We shall denote with $\mathscr{Q}_{1}$ the set of all $T=T_{\tau} \in \mathscr{G}$ such that: (1) $\tau$ is strictly periodic, and (2) if $n$ is the strict period of $\tau$, there is a set $E \in \mathscr{B}$ $s$-admissible of order $n$ for $\tau$. We have obviously $\mathscr{Q}_{1} \subset \mathscr{P}_{1}$ and $\mathscr{Q}_{1} \subset \mathscr{Q}$.

Remark that in the above two definitions condition (1) is in fact a consequence of condition (2). We included condition (1), however, for the sake of clarity.

The following result will be used later: 
(C) Let $T=T_{\tau} \in \mathscr{P}$ (respectively, $T=T_{\tau} \in \mathscr{Q}$ ) and let $\Delta^{\prime}$ be an almost partition of $X$. There are then $E_{1} \in \mathscr{B}, \ldots, E_{k} \in \mathscr{B}$ and $n_{1} \in N^{*}, \cdots, n_{k} \in N^{*}$ such that: $\left(E_{1}, \cdots, E_{k}\right)$ is an admissible (respectively, s-admissible) system of order $\left(n_{1}, \cdots, n_{k}\right)$ for $\tau$ and $\Delta\left(\tau ;\left(E_{1}, \cdots, E_{k}\right) ;\left(n_{1}, \cdots, n_{k}\right)\right)$ is finer than $\Delta^{\prime}$.

Proof. Let $\left(F_{1}, \cdots, F_{q}\right)$ be an admissible (respectively, $s$-admissible) system of order $\left(m_{1}, \cdots, m_{q}\right)$ for $\tau$. The sequence $\left(Y_{i}\right)_{1 \leqq i \leqq q}$, where $Y_{i}=$ $\bigcup_{0 \leqq s \leqq m_{i-1} \tau^{s}}\left(F_{i}\right)$ constitutes an almost partition of $X$. Note that $\tau\left(Y_{i}\right)=Y_{i}$ for each $1 \leqq i \leqq q$. For every $1 \leqq i \leqq q$ denote with $\Delta_{i}^{\prime}$ the almost partition of $Y_{i}$ induced by $\Delta^{\prime}: \Delta_{i}^{\prime}=\Delta^{\prime} \cap Y_{i}$; let $\Delta_{i}^{\prime \prime}=\mathrm{V}_{0 \leqq s \leqq m_{i-1}} \tau^{s} \Delta_{i}^{\prime}$. Finally let $\left\{E_{i, 1}, \cdots, E_{i, p_{i}}\right\}$ be the almost partition of $F_{i}$ induced by $\Delta_{i}^{\prime \prime}: \Delta_{i}^{\prime \prime} \cap F_{i}=$ $\left\{E_{i, 1}, \cdots, E_{i, p_{i}}\right\}$. The sequence $\left(E_{i, j}\right)_{1 \leqq i \leqq q, 1 \leqq j \leqq p_{i}}$ constitutes an admissible (respectively, $s$-admissible) system of order $\left(n_{i, j}\right)_{1 \leqq i \leqq q, 1 \leqq j \leqq p_{i}}$ (here $n_{i, j}=m_{i}$ for all $1 \leqq j \leqq p_{i}$ ) for $\tau$ with the desired property.

Definition 5. Let $G \subset X, H \subset X$ be Lebesgue measurable sets with $\mu(G)>0, \mu(H)>0$. We shall call Lebesgue mapping of $G$ onto $H$ any bijective mapping $\zeta$ of $G$ onto $H$ satisfying the following conditions:

(i) if $E \in \mathscr{B}_{G}$ then $\zeta(E) \in \mathscr{B}_{H}$ and if $F \in \mathscr{B}_{H}$ then $\zeta^{-1}(F) \in \mathscr{B}_{G}$;

(ii) $\mu(\zeta(E))=(\mu(H) / \mu(G)) \cdot \mu(E)$ for any $E \in \mathscr{B}_{G}$.

Remark that $\zeta: G \rightarrow H$ is a Lebesgue mapping if and only if $\zeta^{-1}: H \rightarrow G$ is a Lebesgue mapping.

Let us also remark that given two Lebesgue measurable subsets of $X, G$ and $H$, with $\mu(G)>0$ and $\mu(H)>0$, there exists a Lebesgue mapping of $G$ onto $H$ (use for instance the lemma on p. 74 in [5]).

(D) Let $m>1$ and $\left(G_{i}\right)_{0 \leqq i \leqq m}$ a sequence of Lebesgue measurable subsets of $X$ with $\mu\left(G_{i}\right)>0$ for $0 \leqq i \leqq m$. For each $0 \leqq i<m$, let $\zeta_{i}$ be a Lebesgue mapping of $G_{i}$ onto $G_{i+1}$. Then $\zeta_{m-1} \circ \ldots \circ \zeta_{0}: G_{0} \rightarrow G_{m}$ is a Lebesgue mapping of $G_{0}$ onto $G_{m}$.

Proof. Use induction on $m$.

(E) Let $Y \subset X$ be a Lebesgue measurable set with $\mu(Y)>0$ and let $\left(G_{i}\right)_{0 \leqq i \leqq n-1}$ $(n>2)$ constitute a partition of $Y$. For each $0 \leqq i<n-1$ let $\zeta_{i}$ be a Lebesgue mapping of $G_{i}$ onto $G_{i+1}$. Then: (1) The mapping $\zeta_{n-1}: G_{n-1} \rightarrow G_{0}$ defined by $\zeta_{n-1}=\zeta_{0}^{-1} \circ \ldots \circ \zeta_{n-2}^{-1}$ is a Lebesgue mapping of $G_{n-1}$ onto $G_{0}$. (2) The mapping $\zeta: Y \rightarrow Y$ defined by the equations $\zeta(x)=\zeta_{i}(x)$ for $x \in G_{i}, 0 \leqq i \leqq n-1$, is an automorphism of $Y$ and $G_{0}$ is an s-admissible set of order $n$ for $\zeta$.

Proof. Use proposition (D) above.

In the converse direction we have:

Proposition 1. Let $T_{\zeta} \in \mathscr{Q}$ and let $\left(A_{1}, \cdots, A_{k}\right)$ be an s-admissible system of order $\left(n_{1}, \cdots, n_{k}\right)$ for $\zeta$. Let $1 \leqq i \leqq k$ such that $n_{i}>1$. Then:

(1a) For every $0<s \leqq n_{i}-1$ we have almost everywhere on $\zeta^{s}\left(A_{i}\right)$

$$
\frac{d_{\mu \circ \zeta^{-1}}}{d \mu}(x)=\frac{\mu\left(\zeta^{-1}\left(A_{i}\right)\right)}{\mu\left(\zeta^{s}\left(A_{i}\right)\right)}=\frac{\mu(F)}{\mu(\zeta(F))}, \quad F \subset \zeta^{-1}\left(A_{i}\right), F \in \mathscr{B}, \mu(F)>0 .
$$


(1b) For $s=0$ we have almost everywhere on $A_{i}$

$\frac{d \mu \circ \zeta^{-1}}{d \mu}(x)=\frac{\mu\left(\zeta^{n_{i}-1}\left(A_{i}\right)\right)}{\mu\left(A_{i}\right)}=\frac{\mu(F)}{\mu(\zeta(F))}, \quad F \subset \zeta^{n_{i}-1}\left(A_{i}\right), F \in \mathscr{B}, \mu(F)>0$.

(1c) In particular,

$$
\zeta_{\mid \zeta^{t}\left(A_{i}\right)}: \zeta^{t}\left(A_{i}\right) \rightarrow \zeta^{t+1}\left(A_{i}\right) \quad \text { for } 0 \leqq t<n_{i}-1
$$

and $\zeta_{15^{n_{i}-1}}{ }_{\left(A_{i}\right)}: \zeta^{n_{i}-1}\left(A_{i}\right) \rightarrow A_{i}$ are Lebesgue mappings.

(2) For every $0<s \leqq n_{i}-1, \zeta_{\mid A_{i}}^{s}: A_{i} \rightarrow \zeta^{s}\left(A_{i}\right)$ is a Lebesgue mapping and we have almost everywhere on $\zeta^{s}\left(A_{i}\right)$

$$
\frac{d \mu \circ \zeta^{-s}}{d_{\mu}}(x)=\frac{\mu\left(A_{i}\right)}{\mu\left(\zeta^{s}\left(A_{i}\right)\right)}=\frac{\mu(F)}{\mu\left(\zeta^{s}(F)\right)}, \quad F \subset A_{i}, F \in \mathscr{B}, \mu(F)>0 .
$$

Proof. (1a) Let $0<s \leqq n_{i}-1$. We know that $\left(d_{\mu} \circ \zeta^{-1 / d \mu}\right)(x)$ is equal to a constant $a_{i s}$ almost everywhere on $\zeta^{s}\left(A_{i}\right)$. Let now $F \subset \zeta^{s-1}\left(A_{i}\right), F \in \mathscr{B}$; then $\zeta(F) \subset \zeta^{s}\left(A_{i}\right)$ and we have

$$
\mu(F)=\mu\left(\zeta^{-1}(\zeta(F))\right)=\int_{\zeta(F)} a_{i s} d \mu=a_{i s} \mu(\zeta(F)) .
$$

In particular, for $F=\zeta^{s-1}\left(A_{i}\right)$ we obtain

$$
\mu\left(\zeta^{s-1}\left(A_{i}\right)\right)=a_{i s} \mu\left(\zeta^{s}\left(A_{i}\right)\right)
$$

Thus (1a) is proved.

(1b) can be proved similarly (we need only remark that $\left.\zeta\left(\zeta^{n_{i}-1}\left(A_{i}\right)\right)=A_{i}\right)$.

(1c) follows from (1a) and (1b).

(2) is a consequence of (1c) and proposition (D) above. This completes the proof of Proposition 1.

3. We shall consider on $\mathscr{G}$ the topology $\mathscr{T}$ induced by the strong operator topology of $\mathscr{L}\left(L^{1}, L^{1}\right)$. Let $T_{0} \in \mathscr{G}$. A fundamental system of neighborhoods of $T_{0}$ in $\mathscr{T}$ is given by the collection of all sets of the form

$$
V\left(T_{0} ; \epsilon ; B_{1}, \cdots, B_{n}\right)=\left\{T \in \mathscr{G} \mid\left\|T_{\phi_{B_{i}}}-T_{0} \phi_{B_{i}}\right\| \leqq \epsilon, 1 \leqq i \leqq n\right\},
$$

where $\epsilon>0, n \in N^{*}, B_{1} \in \mathscr{B}, \cdots, B_{n} \in \mathscr{B}^{\prime}$ are arbitrary (for $B \in \mathscr{B}$ we denote by $\phi_{B}$ the characteristic function of the set $B$ ).

If $\Delta=\left\{A_{1}, \cdots, A_{m}\right\}$ is an almost partition of $X$, we shall use the notation $V\left(T_{0} ; \epsilon ; \Delta\right)$ for the neighborhood $V\left(T_{0} ; \epsilon ; A_{1}, \cdots, A_{m}\right)$ of $T_{0}$.

REMarks. (1) Let $T_{0} \in \mathscr{G}$ and $\mathscr{B} \subset \mathscr{B}$ dense in $\mathscr{B}\left({ }^{6}\right)$. A fundamental

\footnotetext{
( 6 For the topology defined by the semi-distance $(E, F) \rightarrow \mu(E \Delta F)$.
} 
system of neighborhoods of $T_{0}$ in $\mathscr{T}$ is given by the collection of all sets of the form $V\left(T_{0} ; \epsilon ; E_{1}, \cdots, E_{n}\right)$, where $\epsilon>0, n \in N^{*}, E_{1} \in \mathscr{E}, \ldots, E_{n} \in \mathscr{E}$ are arbitrary. (2) Let $T_{0} \in \mathscr{G}, \epsilon>0, B_{1}, \cdots, B_{n} \in \mathscr{B}$ and $\Delta^{\prime}$ an almost partition of $X$ finer than the almost partition of $X$ generated by $B_{1}, \ldots, B_{n}$. There is then $0<\epsilon^{\prime} \leqq \epsilon$ such that $V\left(T_{0} ; \epsilon^{\prime} ; \Delta^{\prime}\right) \subset V\left(T_{0} ; \epsilon ; B_{1}, \cdots, B_{n}\right)$.

Proof. (1) Consider the neighborhood $V\left(T_{0} ; \epsilon ; B_{1}, \cdots, B_{n}\right)$ of $T_{0}$ and choose $E_{1} \in \mathscr{E}, \ldots, E_{n} \in \mathscr{E}$ such that $\mu\left(B_{i} \triangle E_{i}\right) \leqq \epsilon / 3,1 \leqq i \leqq n$. We then have

$$
V\left(T_{0} ; \epsilon / 3 ; E_{1}, \cdots, E_{n}\right) \subset V\left(T_{0} ; \epsilon ; B_{1}, \cdots, B_{n}\right) .
$$

In fact, if $T \in V\left(T_{n} ; \epsilon / 3 ; E_{1}, \cdots, E_{n}\right)$ then

$$
\begin{aligned}
\left\|T \phi_{B_{i}}-T_{0} \phi_{B_{i}}\right\| & \leqq\left\|T \phi_{B_{i}}-T \phi_{E_{i}}\right\|+\left\|T \phi_{E_{i}}-T_{0} \phi_{E_{i}}\right\|+\left\|T_{0} \phi E_{i}-T_{0} \phi_{B_{i}}\right\| \\
& \leqq \mu\left(B_{i} \triangle E_{i}\right)+\left\|T \phi_{E_{i}}-T_{0} \phi_{E_{i}}\right\|+\mu\left(E_{i} \triangle B_{i}\right) \\
& \leqq \epsilon / 3+\epsilon / 3+\epsilon / 3=\epsilon
\end{aligned}
$$

and thus $T \in V\left(T_{0} ; \epsilon ; B_{1}, \cdots, B_{n}\right)$.

(2) Assume that $\Delta^{\prime}=\left\{A_{1}, \cdots, A_{p}\right\}$. For each $1 \leqq i \leqq n$, there is a set $J_{i} \subset\{1, \cdots, p\}$ such that $B_{i} \equiv \bigcup_{j \in J_{i}} A_{j}$. Take $\epsilon^{\prime}=\epsilon / p$ and consider the neighborhood $V\left(T_{0} ; \epsilon^{\prime} ; \Delta^{\prime}\right)$ of $T_{0}$. For $T \in V\left(T_{0} ; \epsilon^{\prime} ; \Delta^{\prime}\right)$ we have

$$
\left\|T \phi_{B_{i}}-T_{0} \phi_{B_{i}}\right\| \leqq \sum_{j \in J_{i}}\left\|T \phi_{A_{j}}-T_{0} \phi_{A_{j}}\right\| \leqq p \cdot \epsilon^{\prime}=\epsilon
$$

and hence $V\left(T_{0} ; \epsilon^{\prime} ; \Delta^{\prime}\right) \subset V\left(T_{0} ; \epsilon ; B_{1}, \cdots, B_{n}\right)$.

For further reference we shall collect several useful properties of $\mathscr{T}$ in the following proposition, which we give without proof (see also [5, pp.62-64] and $[8$, p. 123] for the analogy with the measure-preserving case):

Proposition 2. (1) $\mathscr{G}$ endowed with $\mathscr{T}$ is a topological group.

(2) If $\mathscr{E}=\left\{E_{1}, E_{2}, \cdots, E_{k}, \cdots\right\} \subset \mathscr{B}$ is dense in $\mathscr{B}$, then the formula

$$
d(S, T)=\sum_{k=1}^{\infty} \frac{1}{2^{k}}\left(\left\|S_{\phi_{E_{k}}}-T_{\phi_{E_{k}}}\right\|+\left\|S^{-1} \phi_{E_{k}}-T^{-1} \phi_{E_{k}}\right\|\right), \quad S, T \in \mathscr{G},
$$

defines a distance on $\mathscr{G}$ compatible with the topology $\mathscr{T} ; \mathscr{G}$ is complete with respect to the distance $d$, hence in particular $\mathscr{G}$ is a Baire space.

From now on, all the topological considerations concerning $\mathscr{G}$ will refer to the topology $\mathscr{T}$.

We shall make use below of the following unpublished result due to C. E. Linderholm:

(F) LiNDERHOLM'S APPROXIMATION THEOREM. Let $\tau \in \mathscr{A}$ and $\epsilon>0$. There is then $\xi \in \mathscr{A}$ periodic such that $\mu(\{x \mid \xi(x) \neq \tau(x)\}) \leqq \epsilon$. 
We shall now state and prove

Proposition 3. (1) For any $\tau \in \mathscr{A}, \xi \in \mathscr{A}$ and $B \in \mathscr{B}$ we have $\left\|T_{\tau} \phi_{B}-T_{\xi} \phi_{B}\right\| \leqq 2 \mu(B \cap\{x \mid \tau(x) \neq \xi(x)\}) \leqq 2 \mu(\{x \mid \tau(x) \neq \xi(x)\})$.

(2) In particular, $\mathscr{P}$ is dense in $\mathscr{G}$.

Proof. (1) Let $H=\{x \mid \tau(x)=\xi(x)\}$ and $G=\tau(H)=\xi(H)$. For $F \subset G, F \in \mathscr{B}$, we have $\tau^{-1}(F)=\xi^{-1}(F)$ and hence

$$
\int_{F} \frac{d \mu \circ \tau^{-1}}{d \mu}(x) d \mu(x)=\int_{F} \frac{d \mu \circ \xi^{-1}}{\underline{d} \mu}(x) d \mu(x) .
$$

This shows that

$$
\frac{d \mu \circ \tau^{-1}}{d \mu}(x)=\frac{d \mu \circ \xi^{-1}}{d \mu}(x) \text { for almost every } x \in G .
$$

Now $\phi_{B}=\phi_{B \cap H}+\phi_{B \cap C H}$ and $\tau(B \cap H)=\xi(B \cap H) \subset G$. If follows that

$$
T_{\tau} \phi_{B \cap H}=\phi_{\tau(B \cap H)} \cdot \frac{d \mu \circ \tau^{-1}}{d \mu} \equiv \phi_{\xi(B \cap H)} \cdot \frac{d \mu \circ \xi^{-1}}{d \mu}=T_{\xi} \phi_{B \cap H}\left(^{7}\right)
$$

and thus

$$
\left\|T_{\tau} \phi_{B}-T_{\xi} \phi_{B}\right\|=\left\|T_{\tau} \phi_{B \cap C H}-T_{\xi} \phi_{B \cap C H}\right\| \leqq 2 \mu(B \cap \mathbf{C H}) \leqq 2 \mu(\mathrm{CH}) .
$$

Thus (1) is proved; (2) is an immediate consequence of (1) and proposition (F) above. This completes the proof of Proposition 3.

Proposition 4. The set $\mathscr{Q}$ is dense in $\mathscr{G}$.

Proof. The proof will be divided into two parts. We shall show first that (I) The set of all $T=T_{\zeta} \in \mathscr{P}$ for which $\left(d_{\mu} \circ \zeta^{-1}\right) / d \mu \in \mathscr{L}^{\infty}$ is dense in $\mathscr{G}$. By Proposition 3 it will be enough to show that given $\tau \in \mathscr{A}$ periodic and $\epsilon>0$, there is $\zeta \in \mathscr{A}$ periodic such that $\left(d_{\mu} \circ \zeta^{-1}\right) / d_{\mu} \in \mathscr{L}^{\infty}$ and

$$
\mu(\{x \mid \tau(x) \neq \zeta(x)\}) \leqq \epsilon .
$$

Since $\tau$ is periodic, there is $n \in N^{*}$ such that $\tau^{n} \equiv e$ and we may actually assume that $\tau^{n}=e$. We may also assume that $n>1$ (if $n=1$ there is nothing to prove). Consider the measure $\nu=\sum_{0 \leqq i \leqq n-1} \mu \circ \tau^{i} ; \nu$ is absolutely continuous with respect to $\mu$. Hence, given $\epsilon>0$ there is ' $\delta_{\epsilon}>0$ such that the relations $A \in \mathscr{B}$ and $\mu(A) \leqq \delta_{\epsilon}$ imply $\nu(A) \leqq \epsilon$. Choose now $1<N<\infty$ large enough that $\mu\left(\left\{x \mid\left(d_{\mu} \circ \tau^{-1}\right)(x) / d \mu>N\right\}\right) \leqq \delta_{\epsilon}$ and let $A=\left\{x \mid\left(d \mu \circ \tau^{-1}\right)(x) / d \mu>N\right\}$, $B=\bigcup_{0 \leqq i \leqq n-1} \tau^{i}(A)$. We have $\mu(A) \leqq \delta_{t}$, hence $\mu(B) \leqq \nu(A) \leqq \epsilon$; also $\tau(B)$ $=B$. Define now $\zeta$ by the equations

$\left.{ }^{7}\right)$ For $f \in \mathscr{L}^{1}, g \in \mathscr{L}^{1}$, the notation $f \equiv g$ means that $f(x)=g(x)$ for almost every $x \in X$. 


$$
\zeta(x)= \begin{cases}\tau(x) & \text { for } x \in \mathbf{C} B \\ x & \text { for } x \in B\end{cases}
$$

It is easily verified that $\zeta \in \mathscr{A}, \zeta$ is periodic, $\left(d_{\mu} \circ \zeta^{-1} / d_{\mu}\right)(x) \leqq N$ almost everywhere, and that $\mu(\{x \mid \tau(x) \neq \zeta(x)\}) \leqq \epsilon$.

(II) The set $\mathscr{Q}$ is dense in $\mathscr{G}$.

By (I) it will be enough to show that given $T_{. \zeta} \in \mathscr{P}$ for which $\left(d_{\mu} \circ \zeta^{-1}\right) / d_{\mu}$ $\in \mathscr{L}^{\infty}$ and given a neighborhood $W$ of $T_{\zeta}$, there is $T_{\xi} \in \mathscr{Q} \cap W$.

By proposition (C) and Remark (2) above, there are: $\epsilon>0$, sets $E_{1} \in \mathscr{B}$, $\cdots, E_{k} \in \mathscr{B}$ and integers $n_{1} \in N^{*}, \cdots, n_{k} \in N^{*}$ such that $\left(E_{1}, \cdots, E_{k}\right)$ is an admissible system of order $\left(n_{1}, \cdots, n_{k}\right)$ for $\zeta$ and such that

$$
V\left(T_{\zeta} ; \epsilon ; \Delta\left(\zeta ;\left(E_{1}, \cdots, E_{k}\right) ;\left(n_{1}, \cdots, n_{k}\right)\right)\right) \subset W .
$$

We may also assume that $h(x)=\left(d_{\mu} \circ \zeta^{-1} / d_{\mu}\right)(x) \leqq N<\infty$ for every $x \in X$. We shall now define an automorphism $\xi$ with the desired properties.

Let $1 \leqq i \leqq k$.

Suppose first that $n_{i}>1$. Consider the finite sequence $\left(h_{s}\right)_{0 \leqq s \leqq n_{i}-1}$ of functions, where $h_{s}=h \circ \zeta^{s}$ for $0 \leqq s \leqq n_{i}-1$. Choose an almost partition $\Delta_{i}=$ $\left\{H_{i, 1}, \cdots, H_{i, p_{1}}\right\}$ of $E_{i}$ such that for each $1 \leqq j \leqq p_{i}$ the relations $x, y \in H_{i, j}$ imply $\left|h_{s}(x)-h_{s}(y)\right| \leqq \epsilon$ for $0 \leqq s \leqq n_{i}-1$. This means that for each $1 \leqq j \leqq p_{i}$ and each $0 \leqq s \leqq n_{i}-1$ we have

$$
t, u \in \zeta^{s}\left(H_{i, j}\right) \Longrightarrow|h(t)-h(u)| \leqq \epsilon .
$$

Fix now $1 \leqq j \leqq p_{i}$. For each $0 \leqq s<n_{i}-1$, let $\xi_{i, j, s}$ be a Lebesgue mapping of $\zeta^{s}\left(H_{i, j}\right)$ onto $\zeta^{s+1}\left(H_{i, j}\right)$. Define the mapping $\xi_{i, j, n_{i}-1}$ of $\zeta^{n_{i}-1}\left(H_{i, j}\right)$ onto $H_{i, j}$ by the equations: $\xi_{i, j, n_{i}-1}=\xi_{i, j, 0}^{-1}$ if $n_{i}=2$ and $\xi_{i, j, n_{i}-1}=\xi_{i, j, 0}^{-1} \circ \ldots \circ \xi_{i, j, n_{i}-2}^{-1}$ if $n_{i}>2$. By proposition (E), $\xi_{i, j, n_{i}-1}$ is also a Lebesgue mapping.

If $n_{i}=1$, we take $H_{i, 1}=E_{i}\left(p_{i}=1\right.$ in this case $)$ and we define $\zeta_{i, 1,0}(x)=\zeta(x)$ $=x$ for $x \in E_{i}$.

We can at last define the automorphism $\xi$ as follows: $\xi(x)=\xi_{i, j, s}(x)$ if $x \in \zeta^{s}\left(H_{i, j}\right)$ for some $1 \leqq i \leqq k, 1 \leqq j \leqq p_{i}, 0 \leqq s \leqq n_{i}-1$, and $\xi(x)=x$ otherwise. By proposition (E), $\left(H_{i, j}\right)_{1 \leqq i \leqq k, 1 \leqq j \leqq p_{i}}$ is an $s$-admissible system of order $\left(n_{i, j}\right)_{1 \leqq i \leqq k, 1 \leqq j \leqq p_{i}}$ (where $n_{i, j}=n_{i}$ for each $1 \leqq j \leqq p_{i}$ ) for the automorphism $\xi$. Thus $T_{\xi} \in \mathscr{Q}$. It remains to show that $T_{\xi} \in W$. We shall show that $T_{\xi}$ $\in V\left(T_{\zeta} ; \epsilon ; \Delta\left(\zeta ;\left(E_{1}, \cdots, E_{k}\right) ;\left(n_{1}, \cdots, n_{k}\right)\right)\right)$.

Let $1 \leqq i \leqq k$ :

If $n_{i}=1$, then $T_{\xi} \phi_{E_{i}}=\phi_{E_{i}}=T_{\zeta} \phi_{E_{i}}$ and there is nothing to prove.

If $n_{i}>1$ and $0 \leqq s \leqq n_{i}-1$, we have $E_{i} \equiv \bigcup_{1 \leqq j \leqq p_{i}} H_{i, j}$ and hence $\zeta^{s}\left(E_{i}\right)$ $\equiv \bigcup_{1 \leqq j \leqq p_{i}} \zeta^{s}\left(H_{i, j}\right)$. We deduce

$$
\left\|T_{\xi} \phi_{\zeta^{s}\left(E_{i}\right)}-T_{\zeta} \phi_{\zeta}^{s}\left(E_{i}\right)\right\| \leqq \sum_{1 \leqq j \leqq p_{i}}\left\|T_{\xi} \phi_{\zeta^{s}\left(H_{i, j}\right)}-T_{\zeta} \phi_{\zeta^{s}\left(H_{i, j}\right)}\right\| .
$$


Thus it will be enough to prove the inequalities

(**) $\quad\left\|T_{\xi} \phi_{\zeta}{ }^{s}\left(H_{i, j}\right)-T_{\zeta} \phi_{\zeta}{ }^{s}\left(H_{i, j}\right)\right\| \leqq \begin{cases}\epsilon \mu\left(\zeta^{s+1}\left(H_{i, j}\right)\right) & \text { if } 0 \leqq s<n_{i}-1, \\ \epsilon \mu\left(H_{i, j}\right) & \text { if } s=n_{i}-1 .\end{cases}$

CASE $1.0 \leqq s<n_{i}-1$.

In this case we have

$$
\begin{aligned}
& T_{\xi} \phi_{\zeta}{ }^{s}\left(H_{i, j)}=\phi_{\zeta}^{s+1}{ }_{\left(H_{i, j}\right)} \cdot \frac{\mu\left(\zeta^{s}\left(H_{i, j}\right)\right)}{\mu\left(\zeta^{s+1}\left(H_{i, j}\right)\right)},\right. \\
& T_{\zeta \phi_{\zeta}{ }^{s}\left(H_{i, j}\right)}=\phi_{\zeta}^{s+1}{ }_{\left(H_{i, j}\right)} \cdot h
\end{aligned}
$$

and by $(*)$,

$$
\begin{aligned}
& \left\|T_{\xi} \phi_{\zeta}{ }^{s}\left(H_{i, j}\right)-T_{\zeta} \phi_{\zeta}{ }^{s}\left(H_{i, j}\right)\right\| \\
& =\int_{\zeta}^{s+1}\left(H_{i, j}\right)\left|h-\frac{\mu\left(\zeta^{s}\left(H_{i, j}\right)\right)}{\mu\left(\zeta^{s+1}\left(H_{i, j}\right)\right)}\right| d \mu \\
& =\int_{\zeta{ }^{s+1}\left(H_{i, j}\right)}\left|h-\frac{1}{\mu\left(\zeta^{s+1}\left(H_{i, j}\right)\right)} \cdot \int_{s^{s+1}{ }_{\left(H_{i, j}\right)}} h d \mu\right| d_{\mu} \\
& \leqq \epsilon \mu\left(\zeta^{s+1}\left(H_{i, j}\right)\right) .
\end{aligned}
$$

CASE 2. $s=n_{i}-1$.

By a similar computation, using $(*)$, we get

$$
\left\|T_{\xi} \phi_{\zeta}^{n_{i}-1}\left(H_{i, j}\right)-T_{\zeta} \phi_{\zeta}^{n_{i}-1}\left(H_{i, j}\right)\right\| \leqq \epsilon \mu\left(H_{i, j}\right) .
$$

Thus the inequalities $(* *)$ are proved and hence the proposition is completely proved.

4. We shall now prove our main approximation theorem:

Theorem 1. The set $\mathscr{Q}_{1}$ is dense in $\mathscr{G}$. Moreover, given $S \in \mathscr{G}$, a neighborhood $W$ of $S$ and $p \in N^{*}$, there is $T_{\zeta} \in \mathscr{Q}_{1} \cap W$ such that: (1) the strict period $n$ of $\zeta$ is $>p$; (2) there exists an $s$-admissible set $E$ of order $n$ for $\zeta$ such that $\delta(E, \zeta)<1 / p$.

Proof. We may assume without loss of generality that $W$ is an open neighborhood of $S$. By Proposition 4 there is $T_{t} \in \mathscr{Q} \cap W$. By proposition (C) and Remark (2) at the beginning of $\$ 3$, there are: $\epsilon>0$ and an $s$ admissible system $\left(E_{1}, \cdots, E_{k}\right)$ of order $\left(n_{1}, \cdots, n_{k}\right)$ for $\tau$ such that

$$
V\left(T_{r} ; \epsilon ; \Delta\left(\tau ;\left(E_{1}, \cdots, E_{k}\right) ;\left(n_{1}, \cdots, n_{k}\right)\right)\right) \subset W .
$$


Choose an integer $m$ verifying the inequalities:

$$
m>p, \quad \frac{1}{m} \leqq \frac{\epsilon}{2} .
$$

For each $1 \leqq i \leqq k$, divide the set $E_{i}$ into $m$ measurable sets $Y_{i, 1}, \cdots, Y_{i, m}$ of equal measure: $\mu\left(Y_{i, j}\right)=\mu\left(E_{i}\right) / m$, for $1 \leqq j \leqq m$. Denote

$$
G_{i, j, s}=\tau^{s}\left(Y_{i, j}\right), \quad 1 \leqq i \leqq k, \quad 1 \leqq j \leqq m, \quad 0 \leqq s \leqq n_{i}-1 .
$$

Let us now introduce an order relation among the sets $G_{i, j, s}$. We say that $G_{i, j, s}$ precedes $G_{i^{\prime}, j^{\prime}, s^{\prime}}$ if lexicographically $(i, j, s)<\left(i^{\prime}, j^{\prime}, s^{\prime}\right)$ (this means that: either (1) $i<i^{\prime}$, or (2) $i=i^{\prime}$ but $j<j^{\prime}$, or (3) $i=i^{\prime}, j=j^{\prime}$, but $s<s^{\prime}$ ). Remark that this defines a total order. Hence we can arrange the sets $G_{i, j, s}$ in a sequence according to this order: $G_{0}, \cdots, G_{n-1}$, where $G_{0}=G_{1,1,0}, G_{n-1}=G_{k, m, n_{k}-1}$ and $n=m\left(\sum_{1 \leqq i \leqq k} n_{i}\right)$. For each $0 \leqq t<n-1$, let $\zeta_{t}$ be a Lebesgue mapping of $G_{t}$ onto $G_{t+1}$. Define the mapping $\zeta_{n-1}: G_{n-1} \rightarrow G_{0}$ by the equations: $\zeta_{n-1}$ $=\zeta_{0}^{-1}$ if $n=2$ and $\zeta_{n-1}=\zeta_{0}^{-1} \circ \ldots \circ \zeta_{n-2}^{-1}$ if $n>2$. Then $\zeta_{n-1}$ is also a Lebesgue mapping (use proposition (E) above). Define the mapping $\zeta: X \rightarrow X$ by the equations: $\zeta(x)=\zeta_{t}(x)$ if $x \in G_{t}$ for some $0 \leqq t \leqq n-1$, and $\zeta(x)=x$ otherwise. By proposition (E), $\zeta$ is an automorphism and $E=G_{0}$ is an $s$-admissible set of order $n$ for $\zeta$. Thus $T_{\zeta} \in \mathscr{Q}_{1}$. Remark also that the strict period $n$ of $\zeta$ verifies the inequality:

$$
n=m\left(\sum_{1 \leqq i \leqq k} n_{i}\right) \geqq m>p .
$$

Thus (1) is satisfied. On the other hand, we have for the $s$-admissible set $E=G_{0}$ of $\zeta$

$$
\delta(E, \zeta)=\sup _{0 \leqq t \leqq n-1} \mu\left(G_{t}\right)
$$

But each of the sets $G_{t}$ is of the form $G_{i, j, s}=\tau^{s}\left(Y_{i, j}\right)$ for some $1 \leqq i \leqq k$, $1 \leqq j \leqq m, 0 \leqq s \leqq n_{i}-1$, and by (2), Proposition 1 ,

$$
\mu\left(\tau^{s}\left(Y_{i, j}\right)\right)=\frac{1}{m} \mu\left(\tau^{s}\left(E_{i}\right)\right) \leqq \frac{1}{m}<\frac{1}{p} .
$$

Thus $T_{\zeta}$ verifies also (2).

It remains to show that $T_{\zeta} \in W$. For this it will be enough to show that $T_{\zeta} \in V\left(T_{r} ; \epsilon ; \Delta\left(\tau ;\left(E_{1}, \cdots, E_{k}\right) ;\left(n_{1}, \cdots, n_{k}\right)\right)\right)$.

Let $1 \leqq i \leqq k$ :

CASE $1.0 \leqq s<n_{i}-1$.

Remark that $\tau^{s}\left(E_{i}\right)=\bigcup_{1 \leqq j \leqq m} \tau^{s}\left(Y_{i, j}\right)=\bigcup_{1 \leqq j \leqq m} G_{i, j, s}$; using the relations (*) and (1a), Proposition 1, we get 


$$
\begin{aligned}
T_{\zeta} \phi_{\tau}{ }_{\tau}^{s}\left(E_{i}\right) & =\sum_{1 \leqq j \leqq m} T_{\zeta} \phi G_{i, j, s}=\sum_{1 \leqq j \leqq m} \phi_{G_{i, j, s+1}} \cdot \frac{\mu\left(G_{i, j, s}\right)}{\mu\left(G_{i, j, s+1}\right)} \\
& =\sum_{1 \leqq j \leqq m} \phi_{\tau}^{s+1}\left(Y_{i, j}\right) \cdot \frac{\mu\left(\tau^{s}\left(Y_{i, j}\right)\right)}{\mu\left(\tau^{s+1}\left(Y_{i, j}\right)\right)}=\sum_{1 \leqq j \leqq m} \phi_{r}{ }^{s+1}\left(Y_{i, j}\right) \cdot \frac{\mu\left(\tau^{s}\left(E_{i}\right)\right)}{\mu\left(\tau^{s+1}\left(E_{i}\right)\right)} \\
& =\phi_{\tau}{ }^{s+1}\left(E_{i}\right) \frac{\mu\left(\tau^{s}\left(E_{i}\right)\right)}{\mu\left(\tau^{s+1}\left(E_{i}\right)\right)}=T_{\tau} \phi_{\tau}{ }^{s}\left(E_{i}\right) .
\end{aligned}
$$

Hence in this case

$$
\left\|T_{\zeta} \phi_{\tau}{ }^{s}\left(E_{i}\right)-T_{\tau} \phi_{\tau}{ }^{s}\left(E_{i}\right)\right\|=0
$$

CASE 2. $s=n_{i}-1$.

Remark that $\tau^{n_{i}-1}\left(E_{i}\right)=\bigcup_{1 \leqq j \leqq m} \tau^{n_{i}-1}\left(Y_{i, j}\right)=\bigcup_{1 \leqq j \leqq m} G_{i, j, n_{i}-1}$; using (1a), (1b) of Proposition 1 and the relations (*) again, we get

$$
\begin{aligned}
T_{\zeta} \phi_{r}^{n_{i}-1}\left(E_{i}\right) & =\sum_{1 \leqq j \leqq m} T_{\zeta} \phi_{G_{i, j, n_{i}-1}}=\sum_{1 \leqq j<m} T_{\zeta} \phi_{G_{i, j, n_{i}-1}}+T_{\zeta} \phi_{G_{i, m, n_{i}-1}} \\
& =\sum_{1 \leqq j<m} \phi_{G_{i, j+1,0}} \cdot \frac{\mu\left(G_{i, j, n_{i}-1}\right)}{\mu\left(G_{i, j+1,0}\right)}+T_{\zeta} \phi_{G_{i, m, n_{i}-1}} \\
& =\sum_{1 \leqq j<m} \phi_{Y_{i, j+1}} \cdot \frac{\mu\left(\tau^{n_{i}-1}\left(Y_{i, j}\right)\right)}{\mu\left(Y_{i, j+1}\right)}+T_{\zeta} \phi_{\tau}^{n_{i}-1}\left(Y_{i, m}\right) \\
& =\sum_{1 \leqq j<m} \phi_{Y_{i, j+1}} \cdot \frac{\mu\left(\tau^{n_{i}-1}\left(E_{i}\right)\right)}{\mu\left(E_{i}\right)}+T_{\zeta} \phi_{\tau}^{n_{i}-1}\left(Y_{i, m}\right) \\
& =\phi \bigcup_{1<j \leqq m} \bigcup_{Y i, j} \cdot \frac{\mu\left(\tau^{n_{i}-1}\left(E_{i}\right)\right)}{\mu\left(E_{i}\right)}+T_{\zeta} \phi_{\tau} n_{i-1}\left(Y_{i, m}\right)
\end{aligned}
$$

Also,

$$
\begin{aligned}
T_{\tau} \phi_{\tau^{n_{i}-1}\left(E_{i}\right)} & =\phi_{E_{i}} \cdot \frac{\mu\left(\tau^{n_{i}-1}\left(E_{i}\right)\right)}{\mu\left(E_{i}\right)} \\
& =\phi \bigcup_{1<j \leqq m} Y_{i, j} \cdot \frac{\mu\left(\tau^{n_{i}-1}\left(E_{i}\right)\right)}{\mu\left(E_{i}\right)}+\phi_{Y i, 1} \cdot \frac{\mu\left(\tau^{n_{i}-1}\left(E_{i}\right)\right)}{\mu\left(E_{i}\right)} \\
& =\phi \underset{1<j \leqq m}{\bigcup_{1, j}} \cdot \frac{\mu\left(\tau^{n_{i}-1}\left(E_{i}\right)\right)}{\mu\left(E_{i}\right)}+T_{\tau} \phi_{\tau}^{n_{i}-1}\left(Y_{i, 1}\right) .
\end{aligned}
$$

We deduce that 


$$
\begin{aligned}
\| T_{\zeta} \phi_{\tau}^{n_{i}-1}\left(E_{i}\right) & -T_{\tau} \phi_{\tau} n_{i-1}\left(E_{i}\right) \\
& =\| T_{\zeta} \phi_{\tau}^{n_{i}-1}\left(Y_{i, m}\right) \\
& \leqq \mu\left(\tau^{n_{i}-1}\left(Y_{i, m}\right)\right)+\mu\left(\tau_{\tau}^{n_{i}-1}\left(Y_{i, 1}\right)\right. \\
& \left.=\frac{2}{m} \mu\left(\tau_{i, 1}\right)\right)
\end{aligned}
$$

This shows that $T_{\zeta} \in V\left(T_{r} ; \epsilon ; \Delta\left(\tau ;\left(E_{1}, \cdots, E_{k}\right) ;\left(n_{1}, \cdots, n_{k}\right)\right)\right)$ and thus completes the proof of the theorem.

Remark. Concerning the above proof see also [5, p.65] and [8, p. 124], for the measure-preserving case.

\section{PART II}

1. For each $T \in \mathscr{G}$ and $(n, m) \in N^{*} \times N^{*}, n \leqq m$, define the function $T^{(n, m)} \in \mathscr{S}$ by the formula

$$
T^{(n, m)}(x)=\sup _{n \leqq j \leqq m} \frac{T^{j} 1(x)}{j} .
$$

Using the fact that $\mathscr{G}$ is a topological group (see Proposition 2, (1)), one can easily deduce that the mapping $T \rightarrow T^{(n, m)}$ of $\mathscr{G}$ into $\mathscr{S}$ is continuous, for each $(n, m) \in N^{*} \times N^{*}, n \leqq m$.

Theorem 2. (1) For each $k \in N^{*}$ let

$$
\mathscr{N}_{k}=\bigcap_{k \leqq n \leqq m}\left\{T \in \mathscr{G} \mid \rho\left(T^{(n, m)}, 0\right) \leqq \frac{1}{5}\right\} .
$$

The set $\mathscr{N}_{k}$ is closed and nowhere dense in $\mathscr{G}$. (2) The set $\mathscr{N}=\bigcup_{k \in N^{*}} \mathscr{N}_{k}$ is a set of first category in the Baire space $\mathscr{G}$.

Proof. It is enough to prove (1), since (2) is an obvious consequence of (1) (see also Proposition 2, (2)).

Let $k \in N^{*}$. Since the mapping $T \rightarrow T^{(n, m)}$ of $\mathscr{G}$ into $\mathscr{S}$ is continuous for each $(n, m) \in N^{*} \times N^{*}, n \leqq m$, it is obvious that $\mathscr{N}_{k}$ is closed in $\mathscr{G}$. We shall show now that $\mathscr{N}_{k}$ is nowhere dense in $\mathscr{G}$ :

Let $S \in \mathscr{N}_{k}$ and let $V\left(S ; \epsilon ; B_{1}, \ldots, B_{q}\right)$ be a neighborhood of $S$ (here obviously $\epsilon>0$ and $\left.B_{1} \in \mathscr{B}, \ldots, B_{q} \in \mathscr{B}\right)$.

Choose an integer $p$ satisfying the inequalities:

$$
p>k, \quad \frac{1}{p} \leqq \frac{\epsilon}{4}
$$

By Theorem 1 , there is $T_{\zeta} \in \mathscr{Q}_{1} \cap V\left(S ; \epsilon / 2 ; B_{1}, \ldots, B_{q}\right)$ such that: (1) the strict period $n$ of $\zeta$ is $>p$; (2) there exists an $s$-admissible set $A$ of order $n$ for $\zeta$ such that $\delta(A, \zeta)<1 / p$. We may obviously assume also that 


$$
\bigcup_{0 \leqq s \leqq n-1} \zeta^{s}(A)=X
$$

Using $\zeta$ we shall construct a new automorphism $\xi$ such that $T_{\xi}$ $\in V\left(S ; \epsilon ; B_{1}, \cdots, B_{q}\right)$ and $T_{\xi} \notin \mathscr{N}_{k}$. Let

$$
c=\inf _{0 \leqq s \leqq n-1} \frac{\mu(A)}{\mu\left(\zeta^{s}(A)\right)} .
$$

Construct now a partition $\left\{F_{0}, F_{1}, \cdots, F_{r}\right\}(r>1)$ of $A$ such that

$$
\begin{gathered}
\mu\left(F_{0}\right)=\frac{1}{2} \mu(A), \\
\mu\left(F_{j}\right) \leqq \frac{c \mu\left(F_{0}\right)}{n} \cdot \frac{1}{j+1} \quad \text { for } 1 \leqq j \leqq r .
\end{gathered}
$$

This is possible since the series $\sum_{j}(b /(j+1))$ (here $\left.b=\left(c_{\mu}\left(F_{0}\right)\right) / n\right)$ diverges. Note that the sequence $\left(\zeta^{s}\left(F_{j}\right)\right)_{0 \leqq j \leqq r, 0 \leqq s \leqq n-1}$ constitutes a partition of $X$. For each $0 \leqq j \leqq r, 0 \leqq s \leqq n-1$ let

$$
G_{j, s}=\zeta^{s}\left(F_{j}\right)
$$

As before, we can introduce an order relation among the sets $G_{j, s}$ : we say that $G_{j, s}$ precedes $G_{j^{\prime}, s^{\prime}}$ if lexicographically $(j, s)<\left(j^{\prime}, s^{\prime}\right)$ (this means that either $j<j^{\prime}$ or that $j=j^{\prime}$ but $\left.s<s^{\prime}\right)$. This defines a total order. Hence we can arrange the sets $G_{j, s}$ in a sequence according to this order. This sequence consists of $n(r+1)$ elements, the first one being $G_{0,0}=F_{0}$ and the last one $G_{r, n-1}=\zeta^{n-1}\left(F_{r}\right)$. Correspondingly, we shall define a sequence $\left(\xi_{j, s}\right)_{0 \leqq j \leqq r, 0 \leqq s \leqq n-1}$ of Lebesgue mappings as follows:

For $0 \leqq j \leqq r, 0 \leqq s<n-1$, define $\xi_{j, s}=\zeta_{\mid G j, s}$ (by (1c), Proposition 1, $\zeta_{\mid G j, s}$ is a Lebesgue mapping of $G_{j, s}$ onto $G_{j, s+1}$ ).

For $0 \leqq j<r, s=n-1$, let $\xi_{j, n-1}$ be any Lebesgue mapping of $G_{j, n-1}$ onto $G_{j+1,0}$.

The last Lebesgue mapping $\xi_{r, n-1}: G_{r, n-1}=\zeta^{n-1}\left(F_{r}\right) \rightarrow G_{0,0}=F_{0}$ is now uniquely determined by the previous ones, as in (1), proposition (E). Define the automorphism $\xi$ by the equations $\xi(x)=\xi_{j, s}(x)$ if $x \in G_{j, s}$ for some $0 \leqq j \leqq r, 0 \leqq s \leqq n-1$. By (2), proposition (E), $G_{0,0}=F_{0}$ is an $s$-admissible set of order $n(r+1)$ for $\xi$. Remark also that

$$
\{x \mid \xi(x) \neq \zeta(x)\}=\bigcup_{0 \leqq j \leqq r} \zeta^{n-1}\left(F_{j}\right)=\zeta^{n-1}(A) .
$$

We deduce that

$$
\dot{\mu}(\{x \mid \xi(x) \neq \zeta(x)\}) \leqq \mu\left(\zeta^{n-1}(A)\right) \leqq \delta(A, \zeta)<\frac{1}{p} \leqq \frac{\epsilon}{4}
$$


and hence by (1), Proposition 3 , that $T_{\xi} \in V\left(T_{\zeta} ; \epsilon / 2 ; B_{1}, \cdots, B_{q}\right)$. Since $T_{\zeta} \in V\left(S ; \epsilon / 2 ; B_{1}, \cdots, B_{q}\right)$, it follows that $T_{\xi} \in V\left(S ; \epsilon ; B_{1}, \cdots, B_{q}\right)$. Thus the first assertion concerning $T_{\xi}$ is proved. It remains to show that $T_{\xi} \notin \mathscr{N}_{k}$. For this it will be enough to show that:

$(\gamma)$ There is a set $K \in \mathscr{B}, \mu(K)=\frac{1}{2}$ such that for almost every $x \in K$

$$
\sup _{n \leqq t \leqq n(r+1)-1} \frac{T_{\xi}^{t} \phi_{F_{0}}(x)}{t} \geqq 1
$$

In fact, suppose for a moment that $(\gamma)$ is proved. Since $T_{\xi}$ is a positive operator, we deduce from $(\gamma)$ that

$$
T_{\xi}^{(n, n(r+1)-1)}(x) \geqq 1 \text { for almost every } x \in K .
$$

But this implies

$$
\begin{aligned}
\rho\left(T_{\xi}^{(n, n(r+1)-1)}, 0\right) & =\int_{X} \frac{T_{\xi}^{(n, n(r+1)-1)}(x)}{1+T_{\xi}^{(n, n(r+1)-1)}(x)} d \mu(x) \\
& \geqq \int_{K} \frac{T_{\xi}^{(n, n(r+1)-1)}(x)}{1+T_{\xi}^{(n, n(r+1)-1)}(x)} d \mu(x) \geqq \int_{K} \frac{1}{1+1} \quad d_{\mu}=\frac{1}{4}>\frac{1}{5},
\end{aligned}
$$

and since $n>p>k$, this shows that $T_{\xi} \notin \mathscr{N}_{k}$, as asserted.

Let us now prove $(\gamma)$. Let

$$
B=\bigcup_{1 \leqq j \leqq r} F_{r} \text { and } K=\bigcup_{0 \leqq s \leqq n-1} \zeta^{s}(B) .
$$

By condition ( $\alpha$ ) above and by (2), Proposition 1, we have

$$
\mu(K)=\sum_{0 \leqq s \leqq n-1} \mu\left(\zeta^{s}(B)\right)=\sum_{0 \leqq s \leqq n-1} \frac{1}{2} \mu\left(\zeta^{s}(A)\right)=\frac{1}{2} .
$$

Remark also that

$$
\zeta^{s}\left(F_{j}\right)=\xi^{n j+s}\left(F_{0}\right) \quad \text { for } \quad 0 \leqq j \leqq r, \quad 0 \leqq s \leqq n-1
$$

(this formula can be easily verified by induction). It follows that

$$
K=\bigcup_{0 \leqq s \leqq n-1} \zeta^{s}(B)=\bigcup_{0 \leqq s \leqq n-1} \zeta^{s}\left(\bigcup_{1 \leqq j \leqq r} F_{j}\right)=\bigcup_{0 \leqq s \leqq n-1}\left(\bigcup_{1 \leqq j \leqq r} \xi^{n j+s}\left(F_{0}\right)\right) .
$$

For every $0 \leqq s \leqq n-1,1 \leqq j \leqq r$ we have

$$
T_{\xi}^{n j+s} \phi_{F_{0}}=\phi_{\xi}^{n j+s}\left(F_{0}\right) \frac{d \mu \circ \xi^{-(n j+s)}}{d \mu} .
$$

By (2), Proposition 1, and condition $(\beta)$ above, we have almost everywhere on $\xi^{n j+s}\left(F_{0}\right)$ : 


$$
\begin{aligned}
\frac{d \mu \circ \xi^{-(n j+s)}}{d \mu}(x) & =\frac{\mu\left(F_{0}\right)}{\mu\left(\xi^{n j+s}\left(F_{0}\right)\right)}=\frac{\mu\left(F_{0}\right)}{\mu\left(\zeta^{s}\left(F_{j}\right)\right.} \\
& =\frac{\mu\left(F_{0}\right)}{\mu\left(F_{j}\right)} \cdot \frac{\mu\left(F_{j}\right)}{\mu\left(\zeta^{s}\left(F_{j}\right)\right)}=\frac{\mu\left(F_{0}\right)}{\mu\left(F_{j}\right)} \cdot \frac{\mu(A)}{\mu\left(\zeta^{s}(A)\right)} \\
& \geqq \frac{\mu\left(F_{0}\right)}{\mu\left(F_{j}\right)} \cdot c \geqq \frac{n(j+1)}{c} \cdot c=n j+n>n j+s
\end{aligned}
$$

and hence

$$
\frac{T_{\xi}^{n j+s} \phi_{F_{0}}(x)}{n j+s} \geqq 1 \quad \text { almost everywhere on } \xi^{n j+s}\left(F_{0}\right) .
$$

Thus $(\gamma)$ is proved. This completes the proof of Theorem 2 .

2. Let $T=T_{r} \in \mathscr{G}$. We say that $T$ admits a totally $\sigma$-finite invariant measure equivalent to $\mu$ if there is a totally $\sigma$-finite measure $\nu$ on $\mathscr{B}$, invariant under $\tau$ (i.e., satisfying $\nu \circ \tau^{-1}=\nu$ ) and equivalent to $\mu$. We say that the individual ergodic theorem holds for $T$ if for every $f \in \mathscr{L}^{1}$,

$$
\lim _{n \rightarrow \infty}\left(\sum_{0 \leqq j \leqq n-1} T^{j} f(x)\right) / n
$$

exists almost everywhere.

From Theorem 2 we deduce:

Theorem 3. The set of all $T \in \mathscr{G}$ for which the individual ergodic theorem holds is a set of first category in $\mathscr{G}$; hence, the set of all $T \in \mathscr{G}$ which admit a totally $\sigma$-finite invariant measure equivalent to $\mu$ is a set of first category in $\mathscr{G}$.

Proof. Let $T \in \mathscr{G}$ and consider the following assertions:

(a) $T$ admits a totally $\sigma$-finite invariant measure equivalent to $\mu$.

(b) The individual ergodic theorem holds for $T$.

(c) $\lim _{n \rightarrow \infty} T^{n} 1(x) / n=0$ almost everywhere.

(d) $T \in \mathscr{N}$.

(Here $\mathscr{N}=\bigcup_{k \in N^{*}} \mathscr{N}_{k}$ is the set of Theorem 2.) The theorem will be proved if we show that we have the implications: $(a) \Longrightarrow(b) \Longrightarrow(c) \Longrightarrow(d)$.

(a) $\Longrightarrow(\mathrm{b})$. Let $T=T_{\tau} \in \mathscr{G}$ admit a totally $\sigma$-finite measure $\nu$ invariant under $\tau$ and equivalent to $\mu$. Denote by $g$ the Radon-Nikodym derivative of $\mu$ with respect to $\nu$ :

$$
g=\frac{d_{\mu}}{d \nu} .
$$


Then (using Theorem C on p. 163, Theorem B on p. 134 and exercise (2) on p. 136 in [4]) we can write for each $1 \leqq n<\infty$ :

$$
\begin{aligned}
\mu\left(\tau^{-n}(E)\right) & =\int_{\tau^{-n}(E)} g d \nu=\int \phi_{E} \circ \tau^{n} \cdot g d \nu=\int \phi_{E} \cdot g \circ \tau^{-n} d \nu \\
& =\int \phi_{E} \cdot g \circ \tau^{-n} \cdot \frac{1}{g} d \mu=\int_{E} \frac{g \circ \tau^{-n}}{g} d \mu, \quad E \in \mathscr{B} .
\end{aligned}
$$

Thus (see also $[3$, p. 751$]$ ):

$$
\frac{d \mu \circ \tau^{-n}}{d \mu}=\frac{g \circ \tau^{-n}}{g} \quad \text { for each } 1 \leqq n<\infty .
$$

Let now $f \in \mathscr{L}^{1}(\mu)\left({ }^{8}\right)$. Then (use Theorem B on p. 134 in [4]),

$$
f \cdot g \in \mathscr{L}^{1}(\nu)\left({ }^{8}\right)
$$

and for each $1 \leqq n<\infty$

$$
\frac{\sum_{j=0}^{n-1} T^{j} f(x)}{n}=\frac{1}{g(x)} \cdot \frac{\sum_{j=0}^{n-1} f\left(\tau^{-j}(x)\right) \cdot g\left(\tau^{-j}(x)\right)}{n} .
$$

We deduce that $\lim _{n \rightarrow \infty}\left(\sum_{0 \leqq j \leqq n-1} T^{j} f(x)\right) / n$ exists $\nu$-almost everywhere (since $\tau^{-1}$ preserves the measure $\nu$ ) and hence $\mu$-almost everywhere (since $\mu$ and $\nu$ are equivalent). Thus (a) $\Longrightarrow($ b) is proved.

(b) $\Longrightarrow$ (c) is an immediate consequence of the well-known fact that $\lim _{n \rightarrow \infty}\left(a_{n} / n\right)=0$ for a sequence $\left(a_{n}\right)_{1 \leqq n<\infty}$ of real numbers which converges Cesàro to $a$ limit.

(c) $\Longrightarrow(\mathrm{d})$. Let $T \in \mathscr{G}$ be such that $\lim _{n \rightarrow \infty}\left(T^{n} 1(x) / n\right)=0$ almost everywhere. Then $\sup _{1 \leqq j<\infty}\left(T^{j} 1(x) / j\right)$ is finite almost everywhere, and we may actually assume that it is finite everywhere. Thus $F_{n}=\sup _{n \leq j<\infty}\left(T^{j} 1 / j\right) \in \mathscr{S}$ for each $1 \leqq n<\infty$ and $\lim _{n \rightarrow \infty} F_{n}(x)=0$ almost everywhere. It follows that $\lim _{n \rightarrow \infty} \rho\left(F_{n}, 0\right)=0$; there is then $k \in N^{*}$ such that $\rho\left(F_{k}, 0\right) \leqq 1 / 5$. We deduce:

$$
\begin{aligned}
k \leqq n \leqq m & \Longrightarrow T^{(n, m)}(x) \leqq F_{k}(x) \quad \text { for all } x \in X \\
& \Longrightarrow \rho\left(T^{(n, m)}, 0\right) \leqq \rho\left(F_{k}, 0\right) \leqq \frac{1}{5} .
\end{aligned}
$$

This shows that $T \in \mathscr{N}_{k}$ and thus (c) $\Longrightarrow$ (d) is proved. Hence the theorem is completely proved.

REMARK. In connection with Theorem 3, see [2], [6], [10].

( $\left.{ }^{\varnothing}\right)$ To avoid ambiguity, we denote here with $\mathscr{L}^{1}(\mu)$ and $\mathscr{L}^{1}(\nu)$ the spaces of $\mu$-integrable and $\nu$-integrable functions, respectively. 


\section{BibLIOGRAPHY}

1. S. Banach, Théorie des opérations linéaires, Monogr. Mat., Tom. I, Warsaw, 1932.

2. R. V. Chacon, A class of linear transformations, Proc. Amer. Math. Soc. 15 (1964), 560-564.

3. P. R. Halmos, Invariant measures, Ann. of Math. (2) 48 (1947), 735-754.

4. _ Measure theory, Van Nostrand, New York, 1950.

5. L Lectures on ergodic theory, Mathematical Society of Japan, 1956.

6. __ Recent progress in ergodic theory, Bull. Amer. Math. Soc. 67 (1961), 70-80.

7. A. Ionescu Tulcea, Un théorème de catégorie dans la théorie ergodique, C. R. Acad. Sci. Paris 257 (1963), 18-20.

8. K. Jacobs, Neuere Methoden und Ergebnisse der Ergodentheorie, Springer-Verlag, Berlin, 1960.

9. J. Lamperti, On the isometries of certain function-spaces, Pacific J. Math. 8 (1958), 459-466.

10. D. S. Ornstein, On invariant measures, Bull. Amer. Math. Soc. 66 (1960), 297-300.

\section{University of Pennsylvania,}

Philadelphia, Pennsylvania 